\title{
Pregnancy depends on a delicate balance of immune activation and regulation
}

\author{
Shigeru Saito ${ }^{1 *} \mathbb{D}$, Akitoshi Nakashima², Tomoko Shima², Sayaka Tsuda ${ }^{2}$ \\ ${ }^{1}$ President, University of Toyama, Toyama 930-8555, Japan \\ ${ }^{2}$ Department of Obstetrics and Gynecology, University of Toyama, Toyama 930-8555, Japan
}

*Correspondence: Shigeru Saito, University of Toyama, 3190 Gofuku, Toyama 930-8555, Japan. s30saito@med.u-toyama.ac.jp Academic Editor: Satish Kumar Gupta, Indian Council of Medical Research, India

Received: September 2, 2021 Accepted: November 15, 2021 Published: December 31, 2021

Cite this article: Saito S, Nakashima A, Shima T, Tsuda S. Pregnancy depends on a delicate balance of immune activation and regulation. Explor Immunol. 2021;1:461-78. https://doi.org/10.37349/ei.2021.00031

\begin{abstract}
It is well recognized that immune tolerance is important to prevent semiallografted fetuses from rejection by maternal immunocompetent cells; however, immune activation also plays an important role in placental development and fetal growth. Basic and clinical studies have shown that an imbalance between immune activation and regulation can lead to implantation failure, miscarriage, and preeclampsia. Here, the balance between immunostimulation and immunoregulation in reproduction will be reviewed.
\end{abstract}

\section{Keywords}

Cytokine, immune activation, implantation failure, miscarriage, preeclampsia, tolerance

\section{Introduction}

In mammals, fetal (paternal) antigen-specific tolerance is induced by regulatory $\mathrm{T}$ (Treg) cells (Tregs) and other immunosuppressive cells to protect semiallografted fetuses from immunological attack, and pregnancy is maintained. However, immunocompetent cells recognize the fetus and are activated, and various cytokines produced by these cells also play important roles in implantation, trophoblast proliferation and differentiation, placentation, and fetal development. This special issue is in memory of Dr. Gerard Chaouat who passed away. For this reason, we would like to look back on the 40 years of discussions with him and also touch on the historical background of reproductive immunology.

\section{Evolution of reproduction and issues to be solved in mammalian reproduction}

Ovulation occurs in water in fish and amphibians, and eggs are fertilized in water with sperm. As reptiles and birds came to live on land, their reproduction changed from fertilization in water to fertilization inside the female body, after which the fertilized eggs are laid. However, there is a risk that the eggs will be preyed upon. Mammals shifted to the most efficient form of reproduction: fertilization inside the body, development of the fetus through the placenta in the uterus, and subsequent delivery. This reproduction is suitable for protecting the fetus from external threats, but the fetus/placenta, which is considered foreign by the 
maternal host, must be tolerated. Two strategies can be used to solve this problem. The first is to eliminate the diversity of major histocompatibility antigens, which are transplantation antigens, so that the fetus is no longer regarded as a foreign substance. This strategy was adopted by Tasmanian devils. However, they were affected by cancer [1]. Cancer antigens are fragmented into peptides and bound to major histocompatibility molecules. T cells recognize these peptide fragments, and cancer cells are eliminated by T cells; therefore, if major histocompatibility antigens are eliminated, self-mutant tumor cells cannot be eliminated. Tasmanian devils are also territorial and fight by biting each other in order to protect this territory; when tumor cells from tumor bearing Tasmanian devils plant on a wound of a non-tumor bearing Tasmanian devils, tumor cells cannot be recognized as foreign substances, and the tumor grows until the animal eventually dies.

In placental mammals, a system of fetal (paternal) antigen-specific immune tolerance has evolved [2]. This was first demonstrated by Tafuri et al. [3] in 1995. When transgenic CBA mice with a T-cell receptor (TCR) that recognizes the major histocompatibility antigen $\mathrm{H}-2 \mathrm{~K}^{\mathrm{b}}$ were implanted with $\mathrm{C} 57 \mathrm{BL} / 6$-derived tumor cells expressing $\mathrm{H}-2 \mathrm{~K}^{\mathrm{b}}$, the $\mathrm{T}$ cells of these transgenic mice recognized $\mathrm{H}-2 \mathrm{~K}^{\mathrm{b}}$ on the tumor cells and rejected the tumor. On the other hand, when a transgenic female mouse was mated with a C57BL/6 1 mouse and then transplanted with C57BL/6-derived tumor cells, the tumor cells were successfully engrafted during pregnancy, but the tumor cells were rejected within 3 weeks after delivery. In other words, the presence of paternal antigen-specific immune tolerance during pregnancy was experimentally demonstrated, and paternal antigen-specific immune tolerance diminished after delivery.

\section{Changes in the localization of maternal immunocompetent cells during the process from implantation to placentation}

In humans, maternal lymphocytes do not accumulate at the feto-maternal interface when the blastocyst attaches to the endometrial epithelium [4]. However, when trophoblasts break the basement membrane of uterine epithelial cells and invade the stroma, many maternal lymphocytes accumulate around the embryo [4]. This response is similar to that in cervical intraepithelial neoplasia, in which few host lymphocytes accumulate; however, in stage Ia cervical cancer, in which the cancer cells break through the basement membrane and invade the stroma, many lymphocytes accumulate. These changes indicate that the fetus is recognized by the maternal lymphocytes as tumor cells recognized by host lymphocytes.

\section{Do maternal lymphocytes in the decidua (uterus) recognize the fetus and are activated?}

When the authors commenced research in 1980, it was believed that lymphocytes in the uterus are not activated because when maternal lymphocytes are activated, they should attack the fetus and cause miscarriage or implantation failure. However, when mononuclear cells were collected from decidual tissues and analyzed by flow cytometry, lymphocytes were larger in size and had more side scattered light [5]. When lymphocytes were activated, they became blasts and increased in size and side scattered light, which raised the question, "Are maternal lymphocytes activated?" With this, we commenced our research.

In CD16-CD56 ${ }^{\text {bright }}$ natural killer (NK) cells, which account for 70-80\% of the lymphocytes in the decidua, the expression rate of the early activation antigen CD69 was $58.4 \pm 11.3 \%$, which was significantly higher than the expression rate in the peripheral blood $(0.07 \pm 0.03 \%)$ (Table 1) [6]. Human leukocyte antigen (HLA)-DR, which is a late-activation antigen of CD16 CD56 $6^{\text {bright }} \mathrm{NK}$ cells in the decidua, was also significantly higher at $11.3 \pm 4.5 \%$ compared to $0.7 \pm 0.2 \%$ in CD16 CD56 ${ }^{\text {bright }} \mathrm{NK}$ cells in the peripheral blood (Table 1) [6]. These results indicate that $\mathrm{CD} 16 \mathrm{CD} 56^{\text {bright }} \mathrm{NK}$ cells in the decidua are in an activated state. T cells account for $10-20 \%$ of the lymphocytes in the decidua, which are classified into CD4+ $\mathrm{T}$ cells and CD8 ${ }^{+} \mathrm{T}$ cells. As shown in Table 1, the expression of activating antigens, interleukin-2 (IL-2) receptor alpha (IL-2R $\alpha$ ), IL-2R $\beta$, CD69, and HLA-DR, was significantly higher in both $\mathrm{CD} 4^{+}$and $\mathrm{CD} 8^{+} \mathrm{T}$ cells in the decidua than in the peripheral blood [5]. T cells are activated when the CD3/TCR complex recognizes antigens, and the expression level of the CD3/TCR complex is decreased. Therefore, we investigated CD3 and TCR expression levels in T cells in the decidua by flow cytometry. The results showed that the CD3 and TCR expression levels of both $\alpha \beta^{+}$ 
T cells and $\gamma \delta^{+} \mathrm{T}$ cells in the decidua were reduced to approximately two-thirds of those in the peripheral blood [7]. This suggests that both maternal $\alpha \beta^{+} \mathrm{T}$ cells and $\gamma \delta^{+} \mathrm{T}$ cells recognize fetal antigens in the decidua. Antigen-stimulated $\mathrm{T}$ cells differentiate into CD45RO ${ }^{+} \mathrm{CD} 45 \mathrm{RA}-\mathrm{CD} 29^{+}$memory T cells. Both $\mathrm{CD}^{+}$and $\mathrm{CD}^{+}$ $\mathrm{T}$ cells in the decidua are memory type and secrete IL- 2 and IL-4, indicating that $\mathrm{T}$ cells in the decidua are in an activated state [8]. In other words, we demonstrated for the first time that maternal $\mathrm{T}$ cells recognize the fetus and are activated at the feto-maternal interface. We also reported that activated lymphocytes secrete a variety of cytokines that contribute to the proliferation and differentiation of trophoblasts $[9,10]$. Since CD16 CD56 ${ }^{\text {bright }} \mathrm{NK}$ cells in the decidua also express activating antigens, they may produce a variety of cytokines. Previous reports have shown that CD16 CD56 ${ }^{\text {bright }}$ NK cells produce granulocyte colony-stimulating factor (G-CSF), granulocyte macrophage colony-stimulating factor (GM-CSF), macrophage colony-stimulating factor (M-CSF), tumor necrosis factor (TNF)- $\alpha$ (inflammatory cytokine), interferon (IFN) $\gamma$ [T helper 1 (Th1) cytokine and plays an important role in spiral artery remodeling], leukemia inhibitory factor (LIF) (essential cytokine for implantation), IL-8 (inflammation and angiogenesis), vascular endothelial growth factor (VEGF)-A (angiogenesis), angiopoietin-1 (Ang1) (angiogenesis), Ang2 (angiogenesis), transforming growth factor (TGF)- $\beta 1$ (immunoregulatory cytokine), pleiotrophin (fetal growth promoting factor), and osteoglycin (fetal growth promoting factor) [10-13]. G-CSF, GM-CSF, and M-CSF act on trophoblast differentiation and DNA synthesis [9, 14, 15]; IL-8, VEGF-A, Ang1, Ang2, and TGF- $\beta$ act on angiogenesis; and IFN $\gamma$ acts on uterine spiral artery remodeling [16]. In both cases, CD16 CD56 ${ }^{\text {bright }} \mathrm{NK}$ cells play an important role in maintaining pregnancy. Furthermore, Fu et al. [13] reported that Ig-like transcript 2 (ILT2) expressed on uterine NK cells recognize HLA-G expressed on extravillous trophoblasts (EVTs) and secrete pleiotrophin and osteoglycin, which promote fetal growth, while ILT2 on CD16 CD56 $^{\text {bright }} \mathrm{CD} 49 \mathrm{a}^{+}$Eomes $^{+} \mathrm{NK}$ cells recognize HLA-G expressed on EVT. Gamliel et al. [17] also reported that NKG2C and leukocyte immunoglobulin-like receptor subfamily B member 1(LILRB1) expression on CD16 CD56 $6^{\text {bright }}$ NK cells were upregulated in the second and subsequent pregnancies compared to the first pregnancy, recognizing HLA-E and HLA-G expressed on EVT, increasing IFN $\gamma$ production to promote uterine spiral artery remodeling, and increasing VEGF production to promote angiogenesis. Thus, both $\mathrm{T}$ cells and NK cells in the pregnant uterus recognize the fetus, secrete cytokines that promote placental formation, angiogenesis, and fetal growth, and play an important role in the maintenance of pregnancy.

Table 1. Expression of activation markers on decidual and peripheral blood $C D 4^{+} T$ cells, $C D 8^{+} T$ cells, and $C D 16-C D 56^{\text {bright }}$ NK cells

\begin{tabular}{lll}
\hline & $\begin{array}{l}\text { Decidua } \\
\text { \% of positive cells } \\
\text { (mean } \pm \text { SD) }\end{array}$ & $\begin{array}{c}\text { Peripheral blood } \\
\text { of positive cells } \\
\text { (mean } \pm \text { SD) }\end{array}$ \\
\hline CD4 $^{+}$T cells & $26.9 \pm 5.0$ & $20.5 \pm 5.2$ \\
IL-2Ra (CD25) & $8.6 \pm 4.6$ & $1.5 \pm 0.5$ \\
IL-2Rß (CD122) & $58.3 \pm 12.7$ & $0.9 \pm 0.5$ \\
CD69 & $67.7 \pm 14.8$ & $11.8 \pm 4.7$ \\
HLA-DR & & \\
CD8 ${ }^{+}$T cells & $6.2 \pm 2.9$ & $2.5 \pm 1.5$ \\
IL-2Ra (CD25) & $17.7 \pm 7.2$ & $10.6 \pm 3.4$ \\
IL-2Rß (CD122) & $73.3 \pm 14.3$ & $3.5 \pm 0.7$ \\
CD69 & $82.9 \pm 13.5$ & $20.6 \pm 7.4$ \\
HLA-DR & & \\
CD16-CD56 ${ }^{\text {bright }}$ NK cells & $58.4 \pm 11.3$ & $0.07 \pm 0.03$ \\
CD69 & $11.3 \pm 4.5$ & $0.7 \pm 0.2$ \\
\hline HLA-DR & & \\
\hline
\end{tabular}

\section{SD: standard deviation}

Note. Adapted from "Accumulation of $\mathrm{CD} 16-\mathrm{CD} 56^{+}$natural killer cells with high affinity interleukin 2 receptors in human early pregnancy decidua." by Nishikawa K, Saito S, Morii T, Hamada K, Ako H, Narita N, et al. Int Immunol. 1991;3:743-50 (https://academic.oup.com/intimm/article-lookup/doi/10.1093/intimm/3.8.743). @ 1991 Oxford University Press;

"Expression of activation antigens CD69, HLA-DR, interleukin-2 receptor-alpha (IL-2Ra) and IL-2R $\beta$ on T cells of human decidua at an early stage of pregnancy." by Saito S, Nishikawa K, Morii T, Narita N, Enomoto M, Ichijo M. Immunology. 1992;75:710-2 (https://www.ncbi.nlm.nih.gov/pmc/articles/PMC1384855). (c) 1992 Wiley Online Library. 


\section{Induction of fetal (paternal) antigen-specific immune tolerance by Tregs}

How can the fetus avoid rejection when T cells in the decidua are activated? The answers are shown in Table 1. Three years after the authors' report, Sakaguchi et al. [18] demonstrated that CD4+CD25+ (IL-2R $\alpha$ ) T cells had immunosuppressive activity and were identified as Tregs. Subsequent studies revealed that Tregs play a central role in the induction and maintenance of immune tolerance. Later studies then established that Tregs play a central role in the maintenance of allogeneic pregnancy, that is, although fetal-recognizing and activated lymphocytes are present in the decidua, the number of immunosuppressive Tregs increases, preventing excessive immune activation $[19,20]$. Therefore, a balance between immunoactivation and immunoregulation is necessary for the maintenance of pregnancy.

When female mice lacking Tregs were mated with male mice of different genetic backgrounds (allogeneic pregnancy), the fetuses were rejected and miscarriage occurred; however, when mated with mice of the same genetic background (syngeneic pregnancy), no miscarriage occurred (Table 2) [19, 21]. Since all mammals, including humans, undergo allogeneic pregnancy, Tregs are essential for successful pregnancy. Concurrently, the authors reported that Treg levels in peripheral blood were mildly elevated during human pregnancy, that Treg levels in the decidua were markedly increased, and decreased to the same level as in non-pregnant women in cases of miscarriage or recurrent pregnancy loss (RPL) [20].

Table 2. Treg depletion in the implantation period, early pregnancy period, and late pregnancy period

\begin{tabular}{lll}
\hline Treg depletion & Allogenic pregnancy & Syngeneic pregnancy \\
\hline Implantation period & Implantation failure & Normal implantation \\
Early pregnancy period & Miscarriage & Normal pregnancy \\
Late pregnancy period & Normal pregnancy & Normal pregnancy \\
\hline
\end{tabular}

Since the identification of fetal antigen-specific Tregs is not possible in humans, we tested this in mice. DBA/2 mice express the minor lymphocyte stimulating (Mls) Ia antigen, which is recognized by the TCR V $\beta 6$, and paternal antigen-specific Tregs can be identified as CD $4^{+} \mathrm{CD} 25^{+} \mathrm{Foxp} 3^{+} \mathrm{TCR} V \beta 6^{+}$cells. In this study, we crossed BALB/C female mice with DBA/2 male mice and identified Treg-expressing TCR $\mathrm{V} \beta 6$ by flow cytometry [22]. When the Tregs of the mother mouse recognize Mls1, a paternal antigen expressed on the trophoblast, they express Ki67, a cell proliferation marker. In inbred pregnancies in which $\mathrm{BALB} / \mathrm{C}+$ mice were mated with $\mathrm{BALB} / \mathrm{C}{ }^{\Uparrow}$ mice, $\mathrm{V} \beta 6^{+} \mathrm{Ki} 67^{+}$Tregs did not change in the uterus or systemic organs. On the other hand, when $B A L B / C+$ mice were mated with $\mathrm{DBA} / 2{ }^{\circ}$ mice, the number of TCR V $66^{+}{ }^{+} 67^{+}$Tregs increased significantly in the uterus after implantation (4.5 days) and reached the highest level at the late stage of pregnancy [22]. These changes were not observed in the spleen or superficial lymph nodes but were specific to the pregnant uterus [22]. This indicates that Tregs, which recognize paternal antigens expressed in the fetus, proliferate, increase in number in the uterus, and protect the fetus from the attack of maternal immune competent cells. Surprisingly, the uterine regional lymph node, a para-aortic lymph node, had an increased number of Tregs that recognized the paternal antigen $\left(\mathrm{V} \beta 6^{+}\right)$and proliferated $\left(\mathrm{Ki} 67^{+}\right)$one day before implantation [22]. In addition, TCR $\mathrm{V} \beta 6^{+}$Tregs strongly suppressed DNA synthesis in naive T cells compared to TCR V $\beta 6^{-}$Treg in the mixed lymphocyte reaction (MLR), in which maternal $C D 4^{+} \mathrm{CD} 25$-Naive $\mathrm{T}$ cells of $O$ origin were used as responder cells and paternal spleen cells were used as stimulator cells [22], suggesting that TCR V $\beta 6^{+}$Tregs are biologically proven as paternal-antigen specific Tregs. The authors hypothesized that the mechanism by which the number of paternal antigen-specific Tregs increases in the uterine regional lymph nodes one day before implantation may be related to semen exposure. This is because after sexual intercourse, dendritic cells (DCs) in the uterus increase in major histocompatibility complex (MHC)-class II antigens and CD80/86 antigens on their surface and differentiate into mature DCs with antigen-presenting ability. The DCs in the uterus then migrate to the regional lymph nodes of the uterus [23]. 
Semen is composed of seminal plasma and sperm, but seminal plasma is mainly produced in the seminal vesicles, and sperm are produced in the testes. Therefore, we created DBA/ $2 \hat{\varnothing}$ mice with resected seminal vesicles (sperm only) and DBA/2 $\widehat{\gamma}$ mice with ligated vas deferens (seminal plasma only) and crossed them with BALB/C + mice. The number of TCR V $\beta 6^{+} \mathrm{Ki} 67^{+}$Tregs did not increase in the uterine regional lymph nodes 1 day before implantation in the seminal vesicle-resected mice, but did increase in the vas deferens-ligated mice [22] (Table 3). While Robertson et al. [24] already reported that the number of $\mathrm{CD} 4^{+} \mathrm{CD} 25^{+}$Tregs increased in the uterine regional lymph nodes one day before implantation, and this increase was not observed in mice mated with seminal vesicle resected male mice and we confirmed Robertson's data. We have shown that seminal plasma plays an important role in the induction of paternal antigen-specific Tregs by TCR antigen analysis. This is a new point. These results suggest that paternal antigen-specific Tregs are induced by paternal antigens in seminal plasma, and that these cells accumulate in the uterine regional lymph nodes to prepare for implantation. In support of this hypothesis, soluble HLA antigens are contained in semen [25]. It has also been reported that the number of oral sex sessions that induce oral immune tolerance is low in cases of preeclampsia (PE), which is also thought to be a breakdown of immune tolerance to paternal antigens during pregnancy [26]. In addition, seminal plasma contains bioactive TGF- $\beta 1$ and prostaglandin E, which may promote the uptake of paternal antigens by DCs and prostaglandin E, and contribute to paternal antigen-specific immune tolerance [27]. To test this hypothesis, we collected CD11 $\mathrm{c}^{+}$antigen-presenting cells (mostly DCs and some macrophages) from the spleen and uterus of infertile mice, mice mated to DBA $/ 2 \widehat{\diamond}$ mice, and mice mated to DBA $/ 2 \widehat{\diamond}$ mice with resected seminal vesicles [27]. DBA/2 spleen cells were used as stimulator cells and BALB/C spleen cells were used as responder cells, and the antigen-presenting cells were added and cultured in vitro for 8 days to induce paternal antigen-specific Tregs. As a result, $\mathrm{CD} 11^{+}$antigen-presenting cells purified from the uterus of BALB/C $\bigcirc$ mice mated with DBA/2 normal male mice showed an increase in paternal antigen-specific Tregs, whereas CD11 $\mathrm{c}^{+}$antigen-presenting cells purified from the spleen of the same mice had no ability to induce paternal antigen-specific Tregs [27]. CD11 $\mathrm{c}^{+}$antigen-presenting cells purified from the uterus of $\mathrm{BALB} / \mathrm{C}+$ mice mated to seminal vesicle resected ${ }^{\top} \mathrm{mice}$ also had no effect. These results indicate that the paternal antigens contained in seminal plasma are taken up by antigen-presenting cells in the uterus, and the antigen-presenting cells migrate to the uterine regional lymph nodes and present the paternal antigen to $\mathrm{CD} 4^{+} \mathrm{T}$ cells, thereby inducing paternal antigen-specific Tregs in the uterine regional lymph nodes one day before implantation (Figure 1). A meta-analysis reported that intravaginal administration of seminal plasma at the time of egg retrieval or on the day of embryo transfer significantly increased the clinical pregnancy rate [risk ratio (RR) 1.23, 95\% confidence interval (CI): 1.07-1.43, $P=0.003$ ] [28].

Table 3. Paternal antigen-specific Tregs in the uterine regional lymph nodes and uterus mated with normal mice, seminal vesicle resected mice, and deferens-ligated mice

\begin{tabular}{|c|c|c|c|c|c|c|}
\hline & \multirow[t]{3}{*}{ Sperm } & \multirow{3}{*}{$\begin{array}{l}\text { Seminal } \\
\text { plasma }\end{array}$} & \multicolumn{4}{|c|}{ Paternal antigen-specific Tregs } \\
\hline & & & \multicolumn{2}{|c|}{$\begin{array}{l}3.5 \text { days pc } \\
\text { (one day before implantation) }\end{array}$} & \multicolumn{2}{|c|}{$\begin{array}{l}5.5 \text { days pc } \\
\text { (one day after implantation) }\end{array}$} \\
\hline & & & $\begin{array}{l}\text { Uterine } \\
\text { regional } \\
\text { lymph } \\
\text { nodes }\end{array}$ & Uterus & $\begin{array}{l}\text { Uterine } \\
\text { regional } \\
\text { lymph } \\
\text { nodes }\end{array}$ & Uterus \\
\hline Normal mice & + & + & $\uparrow$ & $\rightarrow$ & $\uparrow$ & $\uparrow$ \\
\hline $\begin{array}{l}\text { Seminal vesicle } \\
\text { resected mice }\end{array}$ & + & - & $\rightarrow$ & $\rightarrow$ & $\rightarrow$ & $\rightarrow$ \\
\hline Deferens-ligated mice & - & + & $\uparrow$ & $\rightarrow$ & $\uparrow$ & $\rightarrow$ \\
\hline
\end{tabular}

pc: post-coitus; $\uparrow:$ increase; $\rightarrow$ : no change 


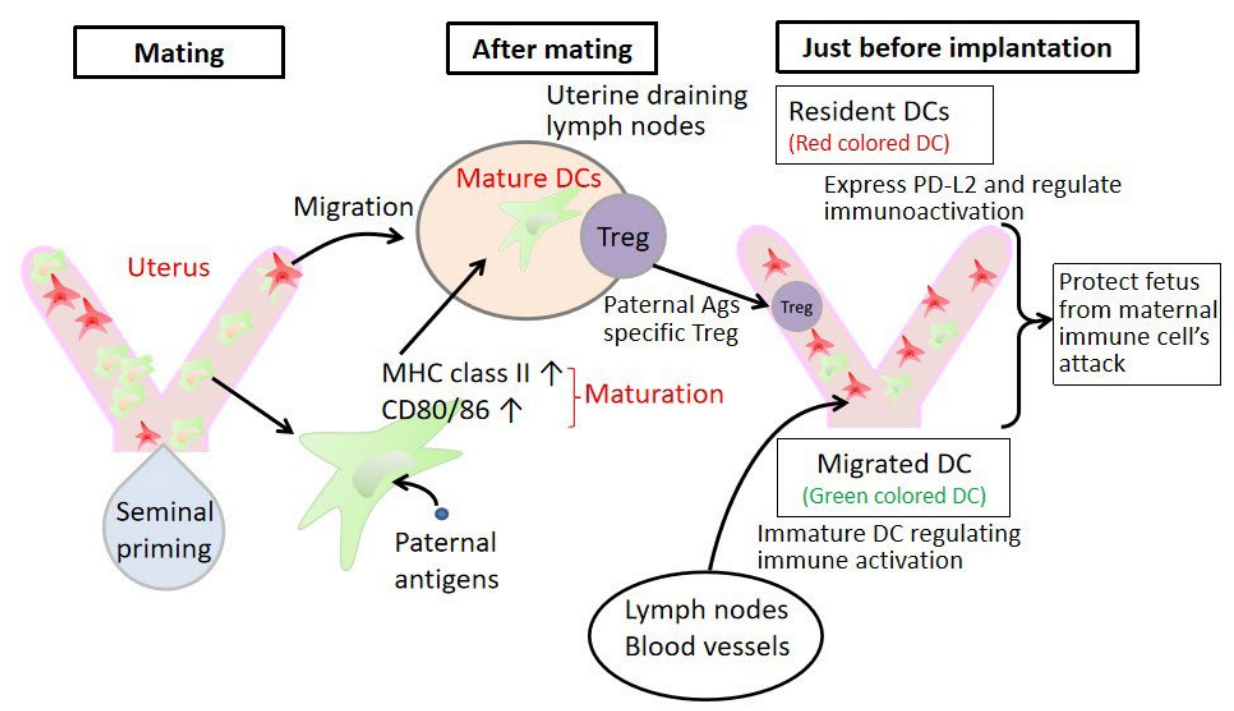

Figure 1. Dynamic changes in the phenotype of uterine DCs. We looked at the migration of DCs using KikGR mice. When the uterus is irradiated with UV light, the cell's color in the uterus turns red. The DCs that migrated from the uterus to other organs afterwards showed red color, which could be identified as DCs originating from the uterus. On the other hand, DCs that have entered the uterus from outside the uterus present a green color and can be distinguished. Using this experimental system, it was presumed that after sexual intercourse, DCs in the uterus mature and increase their antigen-presenting ability. They migrate to the uterine regional lymph nodes and induce paternal antigen-specific Tregs. In addition, some DCs remain in the uterus and express programmed cell death 1 ligand 2 (PD-L2), which contribute to immunoregulation. DCs that enter the uterus from outside the uterus are immature-immunosuppressive DCs. These fine mechanisms allow the fetus to escape the attack of immune cells from the mother

\section{Implantation and immunity}

\section{Tregs and implantation}

Immediately after implantation, maternal lymphocytes accumulate around the embryo and lymphocytes are activated. Administration of anti-CD25 antibody to mice decreases systemic Treg levels for 3 days. In mice, implantation occurs 4.5 days after mating; therefore, when anti-CD25 antibody was administered 2.5 days after mating, 2 days before implantation, the number of Tregs decreased by about $70 \%$ at the time of implantation [22]. When Tregs were depleted by anti-CD25 antibody, implantation failure occurred in the mating of $\mathrm{BALB} / \mathrm{C} q$ and $\mathrm{C} 57 \mathrm{BL} / 6{ }^{\top}$ (allo-pregnancy), but was achieved in the mating of BALB/C $q$ and BALB/C $\widehat{\jmath}$ (syngeneic pregnancy) [21]. Similar findings have been reported previously [29, 30]. It can thus be concluded that Tregs are essential for implantation in allogeneic-pregnant mice. Similar to that in mice, Treg numbers have been found to be reduced in cases of implantation failure in humans [31].

\section{DCs and implantation}

Plaks et al. [32] reported that DCs increase in the uterus during implantation and implantation failure occurs in both allogeneic and syngeneic pregnancies when CD11c ${ }^{+}$DCs are depleted during implantation. Furthermore, they demonstrated that DCs are valuable for angiogenesis, proliferation, and differentiation of decidual stromal cells [32]. This was the first report to show that DCs, innate immune cells, play an important role not only in immunity but also in implantation. Although DCs are cells that migrate between organs in the body, there has been little information on whether the increase in DC numbers during implantation is due to migration from outside the uterus or proliferation in the uterus or whether the uterine DCs change DC subsets. Using KikGR mice, the authors irradiated the uterus with UV light, causing DCs in the uterus to change to a red coloration, and subsequently examined the migration of DCs (Figure 1) [24]. Furthermore, we performed a 6-color analysis of mononuclear cells in the uterus using flow cytometry and analyzed their properties [24]. Immediately after intercourse, DCs in the uterus 
underwent maturation, changed into antigen-presenting cells, and migrated to the uterine regional lymph nodes. Subsequently, a large number of external immature DCs, which induce immune tolerance, flowed into the uterus. Some of the uterine DCs remained in the uterus after coitus, and we found that these DCs expressed PD-L2, which induces immunosuppression (Figure 1, Table 4). In other words, after intercourse, DCs in the uterus mature and migrate to the uterine regional lymph nodes, where they may present paternal antigens to Tregs. Furthermore, we found that immature DCs, which induce immune tolerance, migrated into the uterus from outside the uterus at the time of implantation of the allogeneic embryo (Table 4). In addition, it was observed for the first time that the DCs that stay in the uterus express PD-L2 that induces immunosuppression and change to tolerogenic DCs, which induce immune tolerance; thus, DCs in the uterus can induce tolerance against allogeneic embryos (Figure 1, Table 4). Interestingly, PD-L2 on uterine resident DCs did not change during syngeneic pregnancy (Table 4).

Table 4. Characterization of uterine DC on 3.5 days pc in allogeneic and syngeneic pregnancy

\begin{tabular}{lll}
\hline & Allogeneic pregnancy & Syngeneic pregnancy \\
\hline Immature DC & $\uparrow$ & $\rightarrow$ \\
Mature DC & $\downarrow$ & $\rightarrow$ \\
Migratory DC into uterus & Most of DCs were migratory DC & Not done \\
PD-L2 on uterine resident DC & $\uparrow$ & $\rightarrow$ \\
\hline
\end{tabular}

$\uparrow:$ up-regulation or increase; $\rightarrow$ : no change; $\downarrow$ : down-regulation or decrease

\section{Macrophages and implantation}

CD11b is expressed on M1 macrophages and M2 macrophages. When M1 and M2 macrophages (pan-macrophages) are depleted during implantation using CD11b-DTR mouse, the vascular structure of the corpus luteum is destroyed, progesterone production is reduced; and implantation failure occurs (Table 5) [33]. However, it is unclear whether M1 or M2 macrophages are important for implantation. CD206 is known to be a specific marker for M2 macrophages. Using CD206-diphtheria toxin receptor transgenic (CD206DTX) mice, we depleted CD206 ${ }^{+} \mathrm{M} 2$ macrophages during implantation by administering a small amount of diphtheria toxin [34]. Implantation failure occurred, but there were no histological differences in the corpus luteum, and plasma progesterone levels were also normal. Thus, it is speculated that M1 macrophages are important in luteogenesis. In vitro fertilization (IVF) of eggs derived from mice with depleted $\mathrm{CD} 206^{+} \mathrm{M} 2$ macrophages and transfer of embryos into the uterus of wildtype mice resulted in a normal implantation rate [34]. In other words, the embryos developed normally, even without CD206 ${ }^{+} \mathrm{M} 2$ macrophages. On the other hand, in the endometrium of CD206 ${ }^{+} \mathrm{M} 2$ macrophagedepleted mice, proliferation of endometrial epithelial cells continued [34]. Macrophages are classified into M1 macrophages, M2 macrophages and other macrophages. Since CD11b is expressed on all macrophages, the following can be inferred from the combination of Care's papers and ours. Therefore, CD11 $\mathrm{b}^{+} \mathrm{M} 1$ or $\mathrm{CD} 11 \mathrm{~b}^{+}$other macrophages are necessary for luteogenesis, and CD206 $6^{+} \mathrm{M}$ macrophages are necessary for the endometrial epithelium to stop proliferating and transform into a differentiated endometrial epithelium suitable for implantation (Table 5). Further investigation revealed that when M2 macrophage numbers were reduced, inflammatory cytokines such as TNF- $\alpha$ were produced by M1 macrophages, which increased Wnt $\beta$ expression in the endometrial epithelium, and in turn increased $\beta$-catenin expression; this led to increased fibroblast growth factor (FGF) 18 expression, which proliferated the endometrial epithelium, resulting in implantation failure [34]. Retinoblastoma (RB) protein, encoded by $R b 1$, is a prominent inducer of cell cycle arrest. Akaeda et al. [35] reported that uterine-specific $R b 1$ knockout mice showed implantation failure despite normal progesterone levels in the blood and normal embryo quality, while endometrial epithelial cells showed persistent cell proliferation. They reported implantation failure in this model. Our data and Akaeda's study showed that excessive proliferation of endometrial epithelial cells at the implantation window period induces implantation failure. Endometrial biopsies were performed at the implantation stage in human implantation failure cases. The ratio of M2/M1 macrophage mRNA was significantly lower in the unsuccessful pregnancy group than in the successful pregnancy group in 
the subsequent IVF embryo transfer (IVF-ET) [34]. It is possible that M2 macrophage levels are lower in patients with clinically normal blood progesterone levels but implantation failure occurs due to insufficient differentiation in the secretory phase of the endometrium, determined through endometrial dating or endometrial receptivity analysis.

Table 5. The role of macrophages in implantation failure

\begin{tabular}{|c|c|c|c|c|}
\hline Treatment & $\begin{array}{l}\text { Development of corpus } \\
\text { luteum }\end{array}$ & $\begin{array}{l}\text { Serum } \\
\text { progesterone }\end{array}$ & Endometrium & Implantation \\
\hline Deplete CD11 b+-pan-M $\Phi$ & $\downarrow$ & $\downarrow$ & Proliferation phase & $\downarrow$ \\
\hline Deplete CD206 $-M 2-M \Phi$ & Normal & Normal & $\begin{array}{l}\text { Proliferation phase } \\
\text { (M2-MФs are } \\
\text { essential for the } \\
\text { differentiation of } \\
\text { endometrial epithelial } \\
\text { cells) }\end{array}$ & $\downarrow$ \\
\hline
\end{tabular}

$\downarrow$ : decreased; MФ: macrophage

Considering the treatment of implantation failure from the standpoint of the balance between immune activation and immune regulation

Inflammation is necessary for implantation; however, excessive inflammation can lead to implantation failure [36]. Excessive inflammation can occur when M1 macrophages are dominant over M2 macrophages, when the Th1/Th2 ratio is Th1 dominant, when mature DCs are dominant over immature DCs, and when there are few Tregs (Figure 2). As a treatment for such cases, M1 macrophages can be induced to differentiate into M2 macrophages by administering M-CSF or G-CSF to optimize the M1/M2 macrophage ratio [37, 38]. In some cases, G-CSF has been reported to improve implantation rates in patients with recurrent implantation failure [39]. There is also a report that G-CSF tended to increase clinical pregnancy rates in IVF-ET patients (RR 1.62, 95\% CI: 0.86-3.08). However, no statistically significant differences were observed [40]. For example, administration of G-CSF in patients with decreased M2 macrophages may improve pregnancy rates. The use of immunosuppressive agents such as tacrolimus [41] and anti-TNF- $\alpha$ antibodies [42] may also improve implantation failure in M1 macrophage predominant cases by controlling excessive inflammation. Tregs regulate inflammation and depletion of Tregs at implantation induces implantation failure $[21,29,30]$. If we can develop a method to increase the number of Tregs in the uterus during the implantation period, it may be applied to the treatment of implantation failure. Vitamin D supplementation [43] and high-dose intravenous immunoglobulin (IVIG) therapy [44] may also induce Treg formation and improve implantation failure [45]. Broad spectrum antibiotics may help treat implantation failure in the presence of endometritis [45].

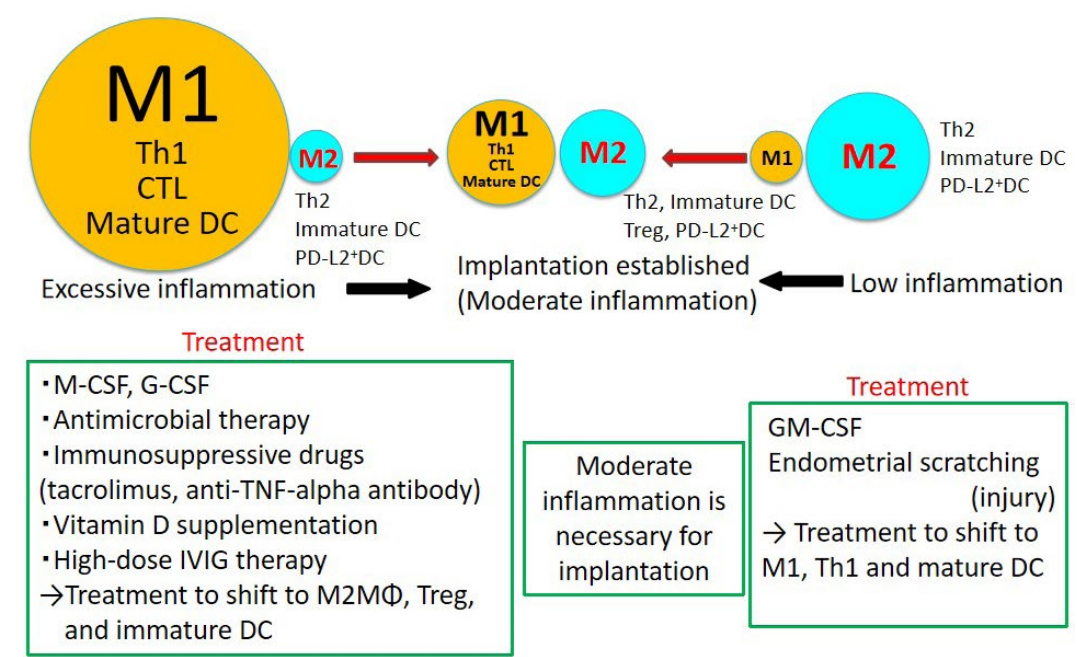

Figure 2. New treatment strategies for implantation failure. Inflammation is necessary for implantation, but too much or too little inflammation may lead to implantation failure. Therefore, we propose that implantation failure be treated separately in cases of excessive inflammation and low inflammation. When the endometrial immune state shows M1 macrophage predominance, Th1 predominance, increased cytotoxic T lymphocytes (CTL) and increased mature DCs, methods to reduce inflammation may be beneficial conversely. In situations where there is little inflammation, methods that activate the immune system may be beneficial 
On the other hand, mechanical stimulation of endometrial cells by the blush technique and induction of differentiation into M1 macrophages by GM-CSF may improve implantation failure in situations where inflammation is low (Figure 2). However, a recent multicenter randomized control study revealed that therapeutic endometrial scratching did not increase the implantation rate [46]. Patient selection is necessary to demonstrate clinical improvement by endometrial scratching.

\section{RPL and immunity}

\section{Immune abnormalities in miscarriage cases with unexplained etiologies}

Human miscarriages occur at a frequency of $10-15 \%$ in the 20 s and 30 s, but the frequency of fetal chromosome abnormalities increases with age, especially in women over 40 years of age, and the frequency of miscarriages increases to $40-60 \%$ [47]. Approximately $60-80 \%$ of miscarriages are due to fetal chromosome abnormalities (mostly aneuploidies), resulting in failure of the fetus to survive. It is well known that chromosomal structural abnormalities of patients and/or partners, uterine malformations, hypothyroidism, and antiphospholipid antibodies are risk factors for RPL, and these risk factors are identified in only 30-40\% of cases [47-49]. In Japan, $65 \%$ of cases have no risk factors identified despite various tests [48]. We hypothesized that miscarriages with unknown risk factors and normal fetal karyotypes are caused by abnormal maternal immunity; therefore, we mainly examined Tregs in the decidua and peripheral blood in such cases.

Immunohistological examination of the localization of Tregs using Foxp3 immunostaining revealed that in normal pregnancies, Tregs were clustered in the decidua basalis near the implantation site. In miscarriages with abnormal fetal karyotypes, Tregs in the decidua basalis were present in the same number as in normal pregnancies. In contrast, the localization of Tregs in the decidua basalis was significantly decreased in miscarriages with normal fetal karyotypes [50]. Interestingly, there was no difference between the three groups in the non-implantation area, that is, changes in Treg localization were observed only around the implantation area in miscarriage cases of normal fetal karyotypes [50].

Foxp3 is a marker of Tregs but is also expressed on activated $\mathrm{T}$ cells that have no immunoregulatory ability [51]. Foxp3 and CD45RA antigen expression can be used to identify effector Tregs with strong immunosuppressive activity (Foxp3 $3^{\text {high }}$ CD45RA), naive Tregs with weak immunosuppressive activity (Foxp3 $^{\text {dim }}$ CD45RA $^{+}$), and activated $\mathrm{T}$ cells (Foxp3 ${ }^{\text {dim }}$ CD45RA), which have no immunosuppressive activity [51]. These Foxp $3^{+}$cells were examined in normal pregnancy, miscarriages with abnormal fetal karyotype, and miscarriages with normal fetal karyotype. It was found that Foxp $3^{\text {high }}$ effector Tregs numbers were significantly decreased and Foxp $3^{\mathrm{dim}}$ activated $\mathrm{T}$ cell numbers were significantly increased in the decidua in miscarriages with normal fetal karyotypes [52]. These results suggest that rejection occurs in normal fetal karyotype miscarriages because the number of effector Tregs, which protect the fetus from maternal immune cells, decreased in the decidua and the number of activated T cells, which attack the fetus, increased in the decidua in humans. On the other hand, peripheral blood effector Treg, naive Treg, and activated T cell populations were the same among the three groups [52], suggesting that abnormal immune status was only present at the feto-maternal interface.

In humans, it is not possible to identify fetal antigen-specific Tregs as in the mouse experimental system. The diversity of TCRs that recognize antigens is known to be more than $2 \times 10^{7}$. Therefore, if Tregs recognize fetal antigens locally, it was presumed that Tregs with the same TCR repertoire sequence would increase clonally because Tregs are stimulated to proliferate by recognition of fetal or paternal antigens. We isolated effector Tregs from peripheral blood and decidua basalis using flow cytometry and performed reverse transcription polymerase chain reaction (RT-PCR) on each cell to sequence TCR sequences from Foxp 3 mRNA $^{+}$cells, which are markers of Tregs. Foxp $3^{+}$Tregs with the same TCR sequence were defined as clonal Tregs and used as surrogate markers for fetal antigen-specific Tregs. The results showed that clonal Tregs were rarely present in peripheral blood but were present in early pregnancy decidua at a median of $4.5 \%$ (1.4-10.8\%) [53]. In addition, all three cases that we were able to analyze in the previous and next pregnancies, respectively, had Tregs with a common TCR sequence between the previous and current pregnancies. Since there is more than $2 \times 10^{7}$ TCR diversity, the likelihood of having the same TCR 
sequence is only $1 / 2 \times 10^{7}$. So it is possible that the previous and current fetuses shared the same major histocompatibility antigens or other minor antigens. It is highly likely that mother Tregs recognize the same major histocompatibility antigens or other minor antigens expressed on trophoblasts and clonally expand. Next, we examined clonal Tregs in decidua and compared them among the three groups of normal pregnancy cases, miscarriages with abnormal fetal karyotypes, and normal fetal karyotypes, and found no differences $[53,54]$. But the frequency of effector Tregs significantly decreased in miscarriage with normal fetal karyotype. In the case of miscarriage with normal fetal karyotype may be caused by anon-specific immunosuppression rather than paternal antigen-specific tolerance.

Cytotoxic $\mathrm{T}$ cells that recognize the paternal antigens attack the fetus resulting in miscarriage in animal model. There is an increased number of effector memory (EM) CD8 ${ }^{+} \mathrm{T}$ cells in the decidua that may attack the fetus in human $[55,56]$. Interestingly, clonal $\mathrm{CD}^{+} \mathrm{T}$ cells are present in EM CD8 ${ }^{+} \mathrm{T}$ cells but are rarely present in naive CD8 ${ }^{+} \mathrm{T}$ cells in humans [55]. Expression of programmed cell death 1 (PD-1) in cytotoxic $\mathrm{T}$ cells results in decreased cytotoxic activity. Kinder et al. [57] reported that PD-1 and lymphocyte activating gene-3 (LAG-3) selectively suppress $\mathrm{CD} 8^{+} \mathrm{T}$ cell cytotoxicity with fetal antigen stimulation in mouse. In cases of normal fetal karyotype miscarriage, the number of clonally expanded EM CD8 ${ }^{+} \mathrm{T}$ cells among CD8 ${ }^{+} \mathrm{T}$ cells was significantly increased by approximately $30 \%$ compared to that in normal pregnancies (15\%). In the early pregnancy period, approximately $80 \%$ of clonal EM CD8 ${ }^{+} \mathrm{T}$ cells are PD-1; therefore, the increased frequency of clonal EM CD8 ${ }^{+} \mathrm{T}$ cells in miscarriages with normal fetal karyotype shows that the frequency of cytotoxic $\mathrm{CD}^{+} \mathrm{T}$ cells against fetal antigens increased. In addition to the decrease in Treg levels, the increase in PD-1 ${ }^{-}$cytotoxic $\mathrm{CD} 8^{+} \mathrm{T}$ cell numbers in the miscarriage cases with normal fetal karyotype was thought to be responsible for the attack on the fetus.

Considering the treatment of RPL from the standpoint of the balance between activation and immune regulation

If Tregs are to be used as a treatment for miscarriage in the future, drug therapy to increase uterus specific Treg pools may be effective [54].

Excessive inflammation has been shown to cause miscarriage not only in mice but also in humans, and vitamin D supplementation [56] and high-dose IVIG therapy [58,59], which increase Treg cell numbers, have been reported to reduce miscarriage in RPL. Anti-TNF- $\alpha$ antibodies have also been reported to reduce miscarriage in cases of RPL [60]. On the other hand, there have been no reports on whether a low inflammatory state can cause RPL; therefore, such RPL is thought to be rare.

\section{PE and immunity}

Immune abnormalities in PE

As we have discussed, the induction of immune tolerance is necessary to maintain pregnancy. The epidemiological risks of PE and miscarriage are different (Table 6). PE is more common in first pregnancies, and the risk is lower in women who have had two or more pregnancies, including previous miscarriages. Even in multiparous women with new partners, the risk of PE was higher in the first pregnancy after a partner change, suggesting that the deficiency of paternal antigen-specific tolerance is associated with the risk of PE. On the other hand, the amount of Tregs in the decidua was lower in miscarriage cases with normal karyotype fetuses, which induced different epidemiological features between PE and RPL.

Seminal plasma induces paternal antigen-specific Tregs; therefore, the increased risk of PE in condom use, short cohabitation, IVF and sperm donation (artificial insemination by donor, AID) pregnancies may be related to inadequate induction of paternal antigen-specific Tregs. In current IVF-ET, embryos are transferred to the uterus without seminal plasma priming, which may result in insufficient induction of paternal antigenspecific Tregs, a lower implantation rate, and a higher risk of PE.

In conventional pregnancies, the fetus is half-self and half-father, but in oocyte donation pregnancies, embryo-donation pregnancies, and hydatidiform moles, the fetus is completely allografted to the maternal 
host. In this case, stronger immune tolerance is required for maintenance of allogeneic pregnancy, and if it is insufficient, the frequency of both PE and miscarriage may increase.

Table 6. Epidemiological risk factors in PE and miscarriage

\begin{tabular}{|c|c|c|}
\hline & PE & Miscarriage \\
\hline \multicolumn{3}{|l|}{ Inadequate induction of tolerance } \\
\hline First pregnancy & $\circ$ & $x$ \\
\hline $\begin{array}{l}\text { The second pregnancy reduces the risk, even if the first pregnancy is a } \\
\text { miscarriage }\end{array}$ & $\circ$ & $x$ \\
\hline First pregnancy with a new partner in multiparous women & $\circ$ & $x$ \\
\hline $\begin{array}{l}\text { More than } 10 \text { years after the last delivery, the risk increases even if adjusted } \\
\text { by age }\end{array}$ & $\circ$ & $x$ \\
\hline IVF-ET & $\circ$ & $x$ \\
\hline \multicolumn{3}{|l|}{ Low or lack of semen exposure } \\
\hline Condom use & $\circ$ & $x$ \\
\hline Short cohabitation & $\circ$ & $x$ \\
\hline Sperm donation pregnancy & $\circ$ & $x$ \\
\hline Egg donation pregnancy & $\circ$ & $\circ$ \\
\hline Embryo donation pregnancy & $\circ$ & $\circ$ \\
\hline \multicolumn{3}{|l|}{ Fetus is a complete allograft to maternal host } \\
\hline Egg donation pregnancy & $\circ$ & $\circ$ \\
\hline Embryo donation pregnancy & $\circ$ & $\circ$ \\
\hline Hydatidiform mole & $\circ$ & $\circ$ \\
\hline
\end{tabular}

o: risk factor; $x$ : not risk factor

From the abovementioned findings, it can be inferred that PE is characterized by a decrease in fetal (paternal) antigen-specific Treg numbers, while miscarriage is characterized by a decrease in overall Treg numbers rather than antigen-specific Treg numbers; however, this has not been fully elucidated scientifically.

The authors were the first to show that Treg reduction in peripheral blood was observed in PE [61]. We also showed, for the first time, that a decrease in Treg levels in peripheral blood was observed in PE [61], and we found a significant decrease in effector Treg levels among Treg levels [62]. Placental bed biopsy of oocyte-donated pregnancies was performed to examine the localization of immunocompetent cells by immunostaining. The numbers of macrophages, $\mathrm{NK}$ cells, $\mathrm{CD} 4^{+} \mathrm{T}$ cells, and Tregs were found to be reduced [63]. Since these cells are involved in the remodeling of uterine spiral arteries by trophoblasts $[12,16,17,64,65]$, cytokeratin 7 (CK7) staining of trophoblasts around spiral arteries showed that remodeling failure of spiral arteries occurred in oocyte-donation pregnancies, which was proven to be a factor contributing to the high risk of PE [63].

To prove the fetal (paternal) antigen-specific tolerance deficiency in PE cases, we examined Treg clonality using the analysis of TCR repertoire in single cell level. The results showed that the number of clonal Tregs in the decidua increased to a median of 20.9\% (15.4-28.1\%) in late normal pregnancy but significantly decreased to a median of 9.3\% (4.4-14.5\%) in PE [53]. These results are consistent with epidemiological findings and demonstrate, for the first time, that one of the etiologies of PE is inadequate induction of fetal (paternal) antigen-specific tolerance. In the future, treatment of PE will require induction and administration of paternal antigen-specific Tregs, which will pose one more hurdle than treatment to increase the overall number of Tregs. The percentage of clonally expanded EM CD8 ${ }^{+} \mathrm{T}$ cells in the decidua basalis of PE cases was approximately $20 \%$, which was not significantly different from the $15 \%$ observed in normal pregnancy cases. In contrast, the PD-1 expression rate in clonally expanded EM CD8 ${ }^{+} \mathrm{T}$ cells was significantly lower in PE cases, with a median of $40 \%$, and $65 \%$ in normal pregnancy cases [56]. In other words, PD-1CTL cells were significantly increased in PE cases compared to those in normal pregnancy cases. This suggests that the fetus is attacked by an increase in PD- $1^{-}$cytotoxic $\mathrm{CD}^{+} \mathrm{T}$ cell numbers, in addition to a decrease in the number of Tregs that recognize fetal antigens. 
The etiology of PE is thought to be shallow placentation due to failure of EVT invasion. In normal pregnancy, EVT invasion extends up to $1 / 3$ of the uterine wall, but in PE, the invasion is shallower, resulting in inadequate remodeling of the spiral artery, which in turn is thought to cause PE. This deep invasion of EVT into uterine muscle wall is specific to primates, making it difficult to model in rodents for experimental use. However, occlusion of uterine artery induces PE-like symptoms in rodents [66]. Using this model, it has been reported that Tregs are decreased in this model, and injection of Tregs improves the PE-like symptoms [67], suggesting that Tregs may be useful for the treatment of PE.

\section{The balance between immune activation and immune regulation in PE}

NK cells recognize HLA-C expressed on trophoblasts by killer cell immunoglobulin-like receptors (KIRs), and KIR AA activates NK cells. Interestingly, the combination of the lack of KIR AA on maternal NK cells and the expression of HLA-C2 in the fetus have been reported to increase the risk of PE [68]. In this combination, NK cells may not be activated and cytokine secretion such as VEGF that promotes angiogenesis may be inadequate. Indeed, VEGF production by T cells and NK cells is decreased in PE [69]. Although there is one study that does not support this report [70], it is interesting to note that the risk of PE increases when the immune system is insufficiently activated.

On the other hand, when PE develops, the Th1/Th2/Th17/Treg balance becomes Th1 cell dominant [71], the number of Th17 cells increases [72], and the number of Tregs decreases [73]. In addition, the number of clonal cytotoxic T cells that attack the fetus increases, and PD-1 expression on these cells also decreases [56]. These results indicate that the immune system is activated in PE. This is contrary to the results of previous studies, which showed that insufficient activation of the immune system is a risk factor for the development of PE [68]. A certain amount of immunoactivation is necessary for pregnancy, but too much activation may lead to PE.

Considering the treatment of PE from the standpoint of the balance between immune activation and immunoregulation

Since seminal plasma exposure is important for paternal antigen-specific Tregs induction, it may be possible to prevent the development of PE if oral contraceptives are recommended instead of condom contraceptive methods, with careful attention to sexually transmitted diseases. However, it is unclear whether this would be effective since there are no such intervention trials.

In preforming IVF-ET, there is no paternal semen exposure in the normal course of the operation. Although it has been reported that intravaginal seminal plasma administration increased the pregnancy rate in IVF-ET [28], whether it suppresses the development of PE has not been reported yet, and we look forward to future results.

If cell therapy using Tregs will be used, it would require the administration of in vitro expanded paternal antigen-specific Tregs is desirable. Because paternal antigen-specific Tregs into PE cases decrease in PE.

PD-1 agonistic antibodies may be effective to manage PE because PD-1 expression is decreased in cytotoxic $\mathrm{T}$ cells, but at present, there are no such clinical trials.

IL-10 administration [74] and anti-TNF- $\alpha$ antibodies [75] may be effective in regulating systemic inflammation at the onset of PE. Although their efficacy has been reported in animal studies, there are no clinical trials at this time, and their efficacy is unknown. Thus, as the immune abnormalities in PE are gradually being identified, immunological treatments may be developed in the near future.

\section{Conclusions}

An immunological study of reproductive phenomena revealed a delicate balance between immune activation and immune regulation. When this balance is disturbed, implantation failure, miscarriage, and PE may occur. Therefore, when considering clinical treatment, it is important to determine whether the patient's immune system is predominantly active or predominantly regulatory and select the optimal treatment based on the 
state of the immune system. We hope that these insights regarding reproductive immunity will be valuable in clinical applications and will lead to the establishment of new therapeutic methods in the future.

\section{Abbreviations}

Ang1: angiopoietin-1

DCs: dendritic cells

EM: effector memory

EVTs: extravillous trophoblasts

FGF: fibroblast growth factor

G-CSF: granulocyte colony-stimulating factor

GM-CSF: granulocyte macrophage colony-stimulating factor

HLA: human leukocyte antigen

IFN: interferon

IL-2: interleukin-2

IL-2R $\alpha$ : interleukin-2 receptor alpha

ILT2: Ig-like transcript 2

IVF: in vitro fertilization

IVF-ET: in vitro fertilization embryo transfer

M-CSF: macrophage colony-stimulating factor

NK: natural killer

PD-1: programmed cell death 1

PD-L2: programmed cell death 1 ligand 2

PE: preeclampsia

RB: retinoblastoma

RPL: recurrent pregnancy loss

TCR: T-cell receptor

TGF: transforming growth factor

Th1: T helper 1

TNF: tumor necrosis factor

Treg: regulatory $\mathrm{T}$

Tregs: regulatory $\mathrm{T}$ cells

VEGF: vascular endothelial growth factor

\section{Declarations}

\section{Author contributions}

SS wrote the paper, and AN consulted on the content of the paper. TS did research on Treg and consulted on the content of the paper. ST submitted materials for the paper.

\section{Conflicts of interest}

The authors declare that they have no conflicts of interest.

Ethical approval

Not applicable. 


\section{Consent to participate}

Not applicable.

\section{Consent to publication}

Not applicable.

\section{Availability of data and materials}

Not applicable.

\section{Funding}

This study was supported by Japan Agency for Medical Research and Development (grant JP18gk0110018 and JP21gk0110047) and Japan Society for the Promotion of Science (KAKENHI grant JP19K09750, 20K09614, 19K18690 and 21K16764). The funders had no role in study design, data collection and analysis, decision to publish, or preparation of the manuscript.

\section{Copyright}

(C) The Author(s) 2021.

\section{References}

1. Woods GM, Howson LJ, Brown GK, Tovar C, Kreiss A, Corcoran LM, et al. Immunology of a transmissible cancer spreading among Tasmanian devils. J Immunol. 2015;195:23-9.

2. Samstein RM, Josefowicz SZ, Arvey A, Treuting PM, Rudensky AY. Extrathymic generation of regulatory T cells in placental mammals mitigates maternal-fetal conflict. Cell. 2012;150:29-38.

3. Tafuri A, Alferink J, Möller P, Hämmerling GJ, Arnold B. T cell awareness of paternal alloantigens during pregnancy. Science. 1995;270:630-3.

4. Boid JB, Hamilton WJ. The human placenta. London and Basingstoke: Machilan Press; 1975. pp. 45-60.

5. Saito S, Nishikawa K, Morii T, Narita N, Enomoto M, Ichijo M. Expression of activation antigens CD69, HLA-DR, interleukin-2 receptor-alpha (IL-2R alpha) and IL-2R beta on T cells of human decidua at an early stage of pregnancy. Immunology. 1992;75:710-2.

6. Nishikawa K, Saito S, Morii T, Hamada K, Ako H, Narita H, et al. Accumulation of CD16 CD56 ${ }^{+}$natural killer cells with high affinity interleukin 2 receptors in human early pregnancy decidua. Int Immunol. 1991;3:743-50.

7. Morii T, Nishikawa K, Saito S, Enomoto M, Ito A, Kurai N, et al. T-cell receptors are expressed but downregulated on intradecidual T lymphocytes. Am J Reprod Immunol. 1993;29:1-4.

8. Saito S, Nishikawa K, Morii T, Narita N, Enomoto M, Ito A, et al. A study of CD45RO, CD45RA and CD29 antigen expression on human decidual T cells in an early stage of pregnancy. Immunol Lett. 1994;40:193-7.

9. Saito S, Saito M, Enomoto M, Ito A, Motoyoshi K, Nakagawa T, et al. Human macrophage colony-stimulating factor induces the differentiation of trophoblast. Growth Factors. 1993;9:11-9.

10. Saito S, Nishikawa K, Morii T, Enomoto M, Narita N, Motoyoshi K, et al. Cytokine production by CD16CD56 ${ }^{\text {bright }}$ natural killer cells in the human early pregnancy decidua. Int Immunol. 1993;5:559-63.

11. Saito S, Kasahara T, Sakakura S, Umekage H, Harada N, Ichijo M. Detection and localization of interleukin-8 mRNA and protein in human placenta and decidual tissues. J Reprod Immunol. 1994;27:161-72.

12. Lash GE, Schiessl B, Kirkley M, Innes BA, Cooper A, Searle RF, et al. Expression of angiogenic growth factors by uterine natural killer cells during early pregnancy. J Leukoc Biol. 2006;80:572-80.

13. Fu B, Zhou Y, Ni X, Tong X, Xu X, Dong Z, et al. Natural killer cells promote fetal development through the secretion of growth-promoting factors. Immunity. 2017;47:1100-13.e6. 
14. Saito S, Fukunaga R, Ichijo M, Nagata S. Expression of granulocyte colony-stimulating factor and its receptor at the fetomaternal interface in murine and human pregnancy. Growth Factors. 1994;10:135-43.

15. Drake BL, Head JR. GM-CSF and CSF-1 stimulate DNA synthesis but not cell proliferation in short-term cultures of mid-gestation murine trophoblast. J Reprod Immunol. 1994;26:41-56.

16. Murphy SP, Tayade C, Ashkar AA, Hatta K, Zhang J, Croy BA. Interferon gamma in successful pregnancies. Biol Reprod. 2009;80:848-59.

17. Gamliel M, Goldman-Wohl D, Isaacson B, Gur C, Stein N, Yamin R, et al. Trained memory of human uterine NK cells enhances their function in subsequent pregnancies. Immunity. 2018;48:951-62.e5.

18. Sakaguchi S, Sakaguchi N, Asano M, Itoh M, Toda M. Immunologic self-tolerance maintained by activated T cells expressing IL-2 receptor alpha-chains (CD25). Breakdown of a single mechanism of self-tolerance causes various autoimmune diseases. J Immunol. 1995;155:1151-64.

19. Aluvihare VR, Kallikourdis M, Betz AG. Regulatory T cells mediate maternal tolerance to the fetus. Nat Immunol. 2004;5:266-71.

20. Sasaki Y, Sakai M, Miyazaki S, Higuma S, Shiozaki A, Saito S. Decidual and peripheral blood CD4 $4^{+} D 25^{+}$ regulatory $\mathrm{T}$ cells in early pregnancy subjects and spontaneous abortion cases. Mol Hum Reprod. 2004;10:347-53.

21. Shima T, Sasaki Y, Itoh M, Nakashima A, Ishii N, Sugamura K, et al. Regulatory T cells are necessary for implantation and maintenance of early pregnancy but not late pregnancy in allogeneic mice. J Reprod Immunol. 2010;85:121-9.

22. Shima T, Inada $K$, Nakashima A, Ushijima A, Ito M, Yoshino O, et al. Paternal antigen-specific proliferating regulatory $\mathrm{T}$ cells are increased in uterine-draining lymph nodes just before implantation and in pregnant uterus just after implantation by seminal plasma-priming in allogeneic mouse pregnancy. J Reprod Immunol. 2015;108:72-82.

23. Yasuda I, Shima T, Moriya T, Ikebuchi R, Kusumoto Y, Ushijima A, et al. Dynamic changes in the phenotype of dendritic cells in the uterus and uterine draining lymph nodes after coitus. Front Immunol. 2020;11:557720.

24. Robertson SA, Guerin LR, Bromfield JJ, Branson KM, Ahlström AC, Care AS. Seminal fluid drives expansion of the $\mathrm{CD} 4^{+} \mathrm{CD} 25^{+} \mathrm{T}$ regulatory cell pool and induces tolerance to paternal alloantigens in mice. Biol Reprod. 2009;80:1036-45.

25. Koelman CA, Coumans AB, Nijman HW, Doxiadis II, Dekker GA, Claas FH. Correlation between oral sex and a low incidence of preeclampsia: a role for soluble HLA in seminal fluid? J Reprod Immunol. 2000;46:155-66.

26. Robertson SA, Ingman WV, O'Leary S, Sharkey DJ, Tremellen KP. Transforming growth factor beta-a mediator of immune deviation in seminal plasma. J Reprod Immunol. 2002;57:109-28.

27. Shima T, Nakashima A, Yasuda I, Ushijima A, Inada K, Tsuda S, et al. Uterine CD11c+ cells induce the development of paternal antigen-specific Tregs via seminal plasma priming. J Reprod Immunol. 2020;141:103165.

28. Crawford G, Ray A, Gudi A, Shah A, Homburg R. The role of seminal plasma for improved outcomes during in vitro fertilization treatment: review of the literature and meta-analysis. Hum Reprod Update. 2015;21:275-84.

29. Darrasse-Jèze G, Klatzmann D, Charlotte F, Salomon BL, Cohen JL. CD4 ${ }^{+} \mathrm{CD} 25^{+}$regulatory/suppressor T cells prevent allogeneic fetus rejection in mice. Immunol Lett. 2006;102:106-9.

30. Zenclussen AC, Gerlof K, Zenclussen ML, Sollwedel A, Bertoja AZ, Ritter T, et al. Abnormal T-cell reactivity against paternal antigens in spontaneous abortion: adoptive transfer of pregnancy-induced $\mathrm{CD} 4^{+} \mathrm{CD} 25^{+} \mathrm{T}$ regulatory cells prevents fetal rejection in a murine abortion model. Am J Pathol. 2005;166:811-22. 
31. Diao LH, Li GG, Zhu YC, Tu WW, Huang CY, Lian RC, et al. Human chorionic gonadotropin potentially affects pregnancy outcome in women with recurrent implantation failure by regulating the homing preference of regulatory T cells. Am J Reprod Immunol. 2017;77:e12618.

32. Plaks V, Birnberg T, Berkutzki T, Sela S, BenYashar A, Kalchenko V, et al. Uterine DCs are crucial for decidua formation during embryo implantation in mice. J Clin Invest. 2008;118:3954-65.

33. Care AS, Diener KR, Jasper MJ, Brown HM, Ingman WV, Robertson SA. Macrophages regulate corpus luteum development during embryo implantation in mice. J Clin Invest. 2013;123:3472-87.

34. Ono Y, Yoshino O, Hiraoka T, Sato E, Fukui Y, Ushijima A, et al. CD206+ M2-like macrophages are essential for successful implantation. Front Immunol. 2020;11:557184.

35. Akaeda S, Hirota Y, Fukui Y, Aikawa S, Shimizu-Hirota R, Kaku T, et al. Retinoblastoma protein promotes uterine epithelial cell cycle arrest and necroptosis for embryo invasion. EMBO Rep. 2021;22:e50927.

36. Mor G, Cardenas I, Abrahams V, Guller S. Inflammation and pregnancy: the role of the immune system at the implantation site. Ann N Y Acad Sci. 2011;1221:80-7.

37. Draijer C, Penke LRK, Peters-Golden M. Distinctive effects of GM-CSF and M-CSF on proliferation and polarization of two major pulmonary macrophage populations. J Immunol. 2019;202:2700-9.

38. Wen Q, Kong Y, Zhao HY, Zhang YY, Han TT, Wang Y, et al. G-CSF-induced macrophage polarization and mobilization may prevent acute graft-versus-host disease after allogeneic hematopoietic stem cell transplantation. Bone Marrow Transplant. 2019;54:1419-33.

39. Davari-Tanha F, Shahrokh Tehraninejad E, Ghazi M, Shahraki Z. The role of G-CSF in recurrent implantation failure: a randomized double blind placebo control trial. Int J Reprod Biomed. 2016;14:737-42.

40. Kamath MS, Kirubakaran R, Sunkara SK. Granulocyte-colony stimulating factor administration for subfertile women undergoing assisted reproduction. Cochrane Database Syst Rev. 2020;1:CD013226.

41. Nakagawa K, Kwak-Kim J, Ota K, Kuroda K, Hisano M, Sugiyama R, et al. Immunosuppression with tacrolimus improved reproductive outcome of women with repeated implantation failure and elevated peripheral blood TH1/TH2 cell ratios. Am J Reprod Immunol. 2015;73:353-61.

42. Winger EE, Reed JL, Ashoush S, El-Toukhy T, Ahuja S, Taranissi M. Degree of TNF- $\alpha /$ IL-10 cytokine elevation correlates with IVF success rates in women undergoing treatment with Adalimumab (Humira) and IVIG. Am J Reprod Immunol. 2011;65:610-8.

43. Paffoni A, Somigliana E, Sarais V, Ferrari S, Reschini M, Makieva S, et al. Effect of vitamin D supplementation on assisted reproduction technology (ART) outcomes and underlying biological mechanisms: protocol of a randomized clinical controlled trial. The "supplementation of vitamin D and reproductive outcome" (SUNDRO) study. BMC Pregnancy Childbirth. 2019;19:395.

44. Abdolmohammadi-Vahid S, Pashazadeh F, Pourmoghaddam Z, Aghebati-Maleki L, Abdollahi-Fard $S$, Yousefi M. The effectiveness of IVIG therapy in pregnancy and live birth rate of women with recurrent implantation failure (RIF): a systematic review and meta-analysis. J Reprod Immunol. 2019;134-135:28-33.

45. Cicinelli E, Matteo M, Tinelli R, Lepera A, Alfonso R, Indraccolo U, et al. Prevalence of chronic endometritis in repeated unexplained implantation failure and the IVF success rate after antibiotic therapy. Hum Reprod. 2015;30:323-30.

46. Olesen MS, Hauge B, Ohrt L, Olesen TN, Roskær J, Bæk V, et al. Therapeutic endometrial scratching and implantation after in vitro fertilization: a multicenter randomized controlled trial. Fertil Steril. 2019;112:1015-21.

47. Dimitriadis E, Menkhorst E, Saito S, Kutteh WH, Brosens JJ. Recurrent pregnancy loss. Nat Rev Dis Primers. 2020;6:98.

48. Morita K, Ono Y, Takeshita T, Sugi T, Fujii T, Yamada H, et al. Risk factors and outcomes of recurrent pregnancy loss in Japan. J Obstet Gynaecol Res. 2019;45:1997-2006. 
49. ESHRE Guideline Group on RPL, Bender Atik R, Christiansen OB, Elson J, Kolte AM, Lewis S, et al. ESHRE guideline: recurrent pregnancy loss. Hum Reprod Open. 2018;2018:hoy004.

50. Inada K, Shima T, Nakashima A, Aoki K, Ito M, Saito S. Characterization of regulatory T cells in decidua of miscarriage cases with abnormal or normal fetal chromosomal content. J Reprod Immunol. 2013;97:104-11.

51. Miyara M, Yoshioka Y, Kitoh A, Shima T, Wing K, Niwa A, et al. Functional delineation and differentiation dynamics of human CD4 ${ }^{+} \mathrm{T}$ cells expressing the FoxP3 transcription factor. Immunity. 2009;30:899-911.

52. Inada K, Shima T, Ito M, Ushijima A, Saito S. Helios-positive functional regulatory T cells are decreased in decidua of miscarriage cases with normal fetal chromosomal content. J Reprod Immunol. 2015;107:10-9.

53. Tsuda S, Zhang X, Hamana H, Shima T, Ushijima A, Tsuda K, et al. Clonally expanded decidual effector regulatory $\mathrm{T}$ cells increase in late gestation of normal pregnancy, but not in preeclampsia, in humans. Front Immunol. 2018;9:1934.

54. Tsuda S, Nakashima A, Shima T, Saito S. New paradigm in the role of regulatory T cells during pregnancy. Front Immunol. 2019;10:573.

55. Tilburgs T, Schonkeren D, Eikmans M, Nagtzaam NM, Datema G, Swings GM, et al. Human decidual tissue contains differentiated $\mathrm{CD}^{+}$effector-memory $\mathrm{T}$ cells with unique properties. J Immunol. 2010;185:4470-7.

56. Morita K, Tsuda S, Kobayashi E, Hamana H, Tsuda K, Shima T, et al. Analysis of TCR repertoire and PD-1 expression in decidual and peripheral CD8+ T cells reveals distinct immune mechanisms in miscarriage and preeclampsia. Front Immunol. 2020;11:1082.

57. Kinder JM, Turner LH, Stelzer IA, Miller-Handley H, Burg A, Shao TY, et al. CD8+ T cell functional exhaustion overrides pregnancy-induced fetal antigen alloimmunization. Cell Rep. 2020;31:107784.

58. Christiansen OB, Kolte AM, Krog MC, Nielsen HS, Egerup P. Treatment with intravenous immunoglobulin in patients with recurrent pregnancy loss: an update. J Reprod Immunol. 2019;133:37-42.

59. Fu J, Li L, Qi L, Zhao L. A randomized controlled trial of etanercept in the treatment of refractory recurrent spontaneous abortion with innate immune disorders. Taiwan J Obstet Gynecol. 2019;58:621-5.

60. Zhao H, Wei X, Yang X. A novel update on vitamin D in recurrent pregnancy loss (Review). Mol Med Rep. 2021;23:382.

61. Sasaki Y, Darmochwal-Kolarz D, Suzuki D, Sakai M, Ito M, Shima T, et al. Proportion of peripheral blood and decidual CD4 ${ }^{+}$CD25 $5^{\text {bright }}$ regulatory T cells in pre-eclampsia. Clin Exp Immunol. 2007;149:139-45.

62. Toldi G, Vásárhelyi ZE, Rigó J Jr, Orbán C, Tamássy Z, Bajnok A, et al. Prevalence of regulatory T-cell subtypes in preeclampsia. Am J Reprod Immunol. 2015;74:110-15.

63. Nakabayashi Y, Nakashima A, Yoshino O, Shima T, Shiozaki A, Adachi T, et al. Impairment of the accumulation of decidual T cells, NK cells, and monocytes, and the poor vascular remodeling of spiral arteries, were observed in oocyte donation cases, regardless of the presence or absence of preeclampsia. J Reprod Immunol. 2016;114:65-74.

64. Robertson SA, Green ES, Care AS, Moldenhauer LM, Prins JR, Hull ML, et al. Therapeutic potential of regulatory T cells in preeclampsia-opportunities and challenges. Front Immunol. 2019;10:478.

65. Staff AC, Fjeldstad HE, Fosheim IK, Moe K, Turowski G, Johnsen GM, et al. Failure of physiological transformation and spiral artery atherosis: their roles in preeclampsia. Am J Obstet Gynecol. 2020; [Epub ahead of print].

66. Burbank F. History of uterine artery occlusion and subsequent pregnancy. AJR Am J Roentgenol. 2009;192:1593-600.

67. Cornelius DC, Amaral LM, Harmon A, Wallace K, Thomas AJ, Campbell N, et al. An increased population of regulatory $\mathrm{T}$ cells improves the pathophysiology of placental ischemia in a rat model of preeclampsia. Am J Physiol Regul Integr Comp Physiol. 2015;309:R884-91. 
68. Hiby SE, Walker JJ, O'shaughnessy KM, Redman CW, Carrington M, Trowsdale J, et al. Combinations of maternal KIR and fetal HLA-C genes influence the risk of preeclampsia and reproductive success. J Exp Med. 2004;200:957-65.

69. Molvarec A, Ito M, Shima T, Yoneda S, Toldi G, Stenczer B, et al. Decreased proportion of peripheral blood vascular endothelial growth factor-expressing $\mathrm{T}$ and natural killer cells in preeclampsia. Am J Obstet Gynecol. 2010;203:567.e1-8.

70. Saito S, Takeda Y, Sakai M, Nakabayashi M, Hayakawa S. The incidence of pre-eclampsia among couples consisting of Japanese women and Caucasian men. J Reprod Immunol. 2006;70:93-8.

71. Saito S, Sakai M, Sasaki Y, Tanebe K, Tsuda H, Michimata T. Quantitative analysis of peripheral blood Th0, Th1, Th2 and the Th1:Th2 cell ratio during normal human pregnancy and preeclampsia. Clin Exp Immunol. 1999;117:550-5.

72. Santner-Nanan B, Peek MJ, Khanam R, Richarts L, Zhu E, Fazekas de St Groth B, et al. Systemic increase in the ratio between Foxp $3^{+}$and IL-17-producing $\mathrm{CD}^{+}{ }^{+} \mathrm{T}$ cells in healthy pregnancy but not in preeclampsia. J Immunol. 2009;183:7023-30.

73. Saito S, Nakashima A, Shima T, Ito M. Th1/Th2/Th17 and regulatory T cell paradigm in pregnancy. Am J Reprod Immunol. 2010;63:601-10.

74. Harmon A, Cornelius D, Amaral L, Paige A, Herse F, Ibrahim T, et al. IL-10 supplementation increases Tregs and decreases hypertension in the RUPP rat model of preeclampsia. Hypertens Pregnancy. 2015;34:291-306.

75. Cunningham MW, Jayaram A, Deer E, Amaral LM, Vaka VR, Ibrahim T, et al. Tumor necrosis factor alpha (TNF- $\alpha$ ) blockade improves natural killer cell (NK) activation, hypertension, and mitochondrial oxidative stress in a preclinical rat model of preeclampsia. Hypertens Pregnancy. 2020;39:399-404. 